\title{
Introduction to Microfabrication Techniques
}

\author{
Rabih Zaouk, Benjamin Y. Park, and Marc J. Madou
}

\begin{abstract}
Summary
The advent of photolithography literally brought about the integrated circuit (IC) revolution of the latter part of the twentieth century. Almost all electronic devices that we use today have one or more ICs inside. Improving lithography techniques led to smaller and smaller transistors, which translated into faster and more efficient computing machines. Photolithography also powered the advent of MicroElectroMechanical Systems (MEMS), which are now starting to become more and more diverse in commercial products from mechanical to biomedical devices, helping to change the way people perceive the applicability of IC technology. In this chapter, we examine basic photolithography techniques and their uses in soft lithography and MEMS.
\end{abstract}

Key Words: Photolithography; microlithography; lithography; soft lithography; microfabrication; MicroElectroMechanical Systems; BioMEMS, microfluidics.

\section{Photolithography: An Overview}

The word lithography (Greek for the words stone [lithos] and to write [gráphein]) refers to a process invented in 1796 by Aloys Senefelder. Senefelder found that stone (he used Bavarian limestone) when properly inked and treated with chemicals could transfer a carved image onto paper (1).

The most widely used form of lithography is photolithography. In this process, a pattern is transferred to a photosensitive polymer (a photoresist) by exposure to a light source through an optical mask. An optical mask usually consists of opaque patterns (usually chrome or iron oxide) on a transparent support (usually quartz) used to define features on a wafer. The pattern in the photoresist is then further transferred to the underlying substrate by subtractive (etching) or additive (deposition) techniques. The combination of accurate alignment of a successive set of photomasks and exposure of these successive 
patterns leads to complex multilayered structures. Photolithography has matured rapidly by continuous improvements in the ability to resolve ever-smaller features. Research in high-aspect-ratio resist features, driven by the field of MicroElectroMechanical Systems (MEMS), is also being actively pursued, as opposed to the essentially two-dimensional processes used traditionally. This is especially important in the fabrication of microfluidic molds.

Photolithography and pattern transfer involve a set of process steps as summarized in Fig. 1. As an example, we use an oxidized silicon ( $\mathrm{Si}$ ) wafer and a negative photoresist to transfer a pattern from a mask to a layer of silicon dioxide. An oxidized wafer (Fig. 1A) is coated with a 1- $\mu \mathrm{m}$-thick negative photoresist layer (Fig. 1B). After exposure (Fig. 1C), the wafer is rinsed in a developing solution or sprayed with a spray developer, which removes the unexposed areas of photoresist and leaves a pattern of bare and photoresistcoated oxide on the wafer surface (Fig. 1D). The resulting photoresist pattern is the negative image of the pattern on the photomask. In a typical next step after development, the wafer is placed in a solution of HF or a mixture of HF and $\mathrm{NH}_{4} \mathrm{~F}$ that attacks the oxide at a much faster rate than the photoresist or the underlying Si (Fig. 1E). The photoresist prevents the oxide underneath from being attacked. Once the exposed oxide has been etched away, the remaining photoresist can be stripped off with a solution that only attacks the photoresist, such as a strong acid (e.g., $\mathrm{H}_{2} \mathrm{SO}_{4}$ ) or an acid-oxidant combination (e.g., piranha, $\mathrm{H}_{2} \mathrm{SO}_{4}: \mathrm{H}_{2} \mathrm{O}_{2}$ ) (Fig. 1F). Other liquid strippers include organic solvent strippers and alkaline strippers (with or without oxidants). The oxidized $\mathrm{Si}$ wafer with etched windows in the oxide (Fig. 1F) now awaits further processing. Recently, photoresists are increasingly being used in applications in which the resist is a permanent part of the final device rather than just a sacrificial layer for patterning the substrate.

\section{Basic Photolithography Techniques}

Each implementation of the photolithographic process has its own specific requirements, but there is a basic common flow of process that are common to most procedures. In this section, we introduce the basic lithography steps of preparation of wafer, resist application, exposure, development, and pattern transfer.

\subsection{Preparation of Wafer}

\subsubsection{Cleaning of Wafer}

Physical contaminants such as dust particles can hinder the lithography process by preventing light from exposing the photoresist or by disturbing the surface uniformity of a coated photoresist. Chemical contaminants may also react with various materials used in the lithography process, creating unwanted effects. 

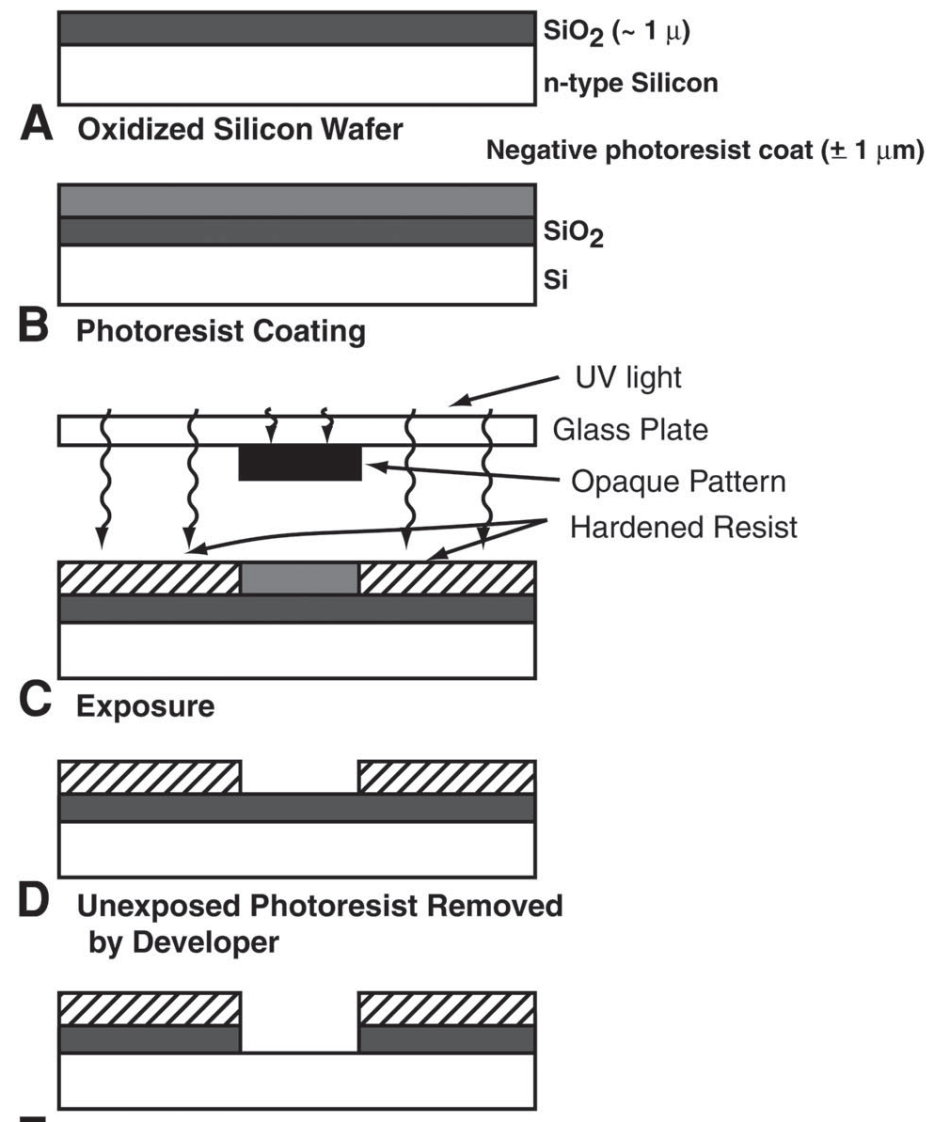

E $\mathrm{SiO}_{2}$ Etched with $\mathrm{HF}$

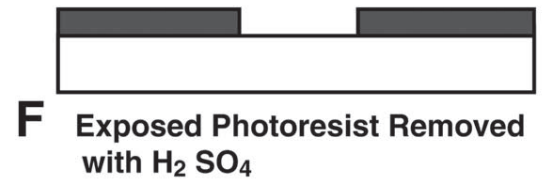

Fig.1. Process flow of basic photolithography followed by pattern transfer. The example uses (A) an oxidized $\mathrm{Si}$ wafer and a negative photoresist system. The process steps are (B) photoresist coating, (C) exposure, (D) development, (E) oxide etching, and $(\mathbf{F})$ resist stripping and oxide etching. These steps are explained in detail in the text. 
A silicon wafer is the most commonly used platform for microfabrication, but there is a trend toward different substrate materials, especially in the field of BioMEMS (see Subheading 4.). A variety of cleaning methods can be used to prepare a wafer for the lithography process. Usually, new wafers do not need further cleaning, because they are cleaned before shipping and are kept in a contamination-free container. If cleaning of the wafer is necessary, a variety of methods (HF dip, RCA1, RCA2, use of piranha, and so on) can be used to remove different types of contaminants. The presence of water or water vapor compromises the adhesion between the photoresist and the wafer. Before the photoresist is applied, a dehydration bake is performed to remove water from the surface of the wafer. Adhesion can be further promoted by applying an adhesion promoter (hexamethyldisilizane) or by roughening the surface of the wafer by plasma etching. This is referred to as wafer priming.

\subsubsection{Oxide Growth}

In many cases, an oxide layer is desired as a mask for subsequent processes (e.g., an etch or an implant process) or as an insulating layer. This is usually achieved by heating a silicon wafer to between 900 and $1150^{\circ} \mathrm{C}$ in a dry or humidified oxygen stream in a tube furnace.

\subsection{Resist Spinning and Soft Bake}

As the first step in the lithography process itself, a thin layer of an organic polymer, a photoresist sensitive to ultraviolet (UV) radiation, is deposited on the oxide surface (see Fig. 1B). The liquid photoresist is dispensed onto a wafer that is held by a vacuum chuck in a resist spinner. The wafer is then spun in one or more steps at precisely controlled speeds. The spin speed (between 1500 and $8000 \mathrm{rpm}$ ) allows the formation of a uniform film. At these speeds, the centrifugal force causes the liquid to flow to the edges, where it builds up until expelled when the surface tension is exceeded. The resulting polymer thickness, $T$, is a function of spin speed, solution concentration, and molecular weight (measured by intrinsic viscosity). The spin curves for various photoresists can be obtained from the manufacturer. The spinning process is of primary importance to the effectiveness of pattern transfer. The quality of the resist coating determines the density of defects transferred to the device under construction.

After spin coating, the resist still contains up to $15 \%$ solvent and may contain built-in stresses. The wafers are therefore soft baked (prebaked) at 75$100^{\circ} \mathrm{C}$ to remove solvents and stress, and to promote adhesion of the resist layer to the wafer. 


\section{Negative and Positive Tone}

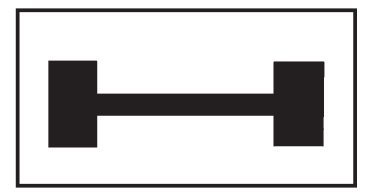

Mask

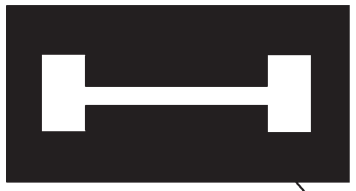

Negative Photoresist

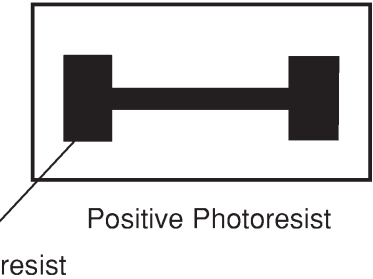

Photoresist

Remaining Pattern after Photoresist Development

Fig. 2. Resulting patterns after exposure and development of a positive- and negative-tone photoresist. The opaque image on the mask is transferred as is onto the positive photoresist. The image is reversed in the case of a negative photoresist.

\subsection{Exposure and Postexposure Treatment}

Pattern transfer onto a photoresist is done by shining light through the mask (see Fig. 1C). One typically uses the g-line $(435 \mathrm{~nm})$ or i-line $(365 \mathrm{~nm})$ of a mercury lamp. In general, the smallest feature that can be printed using projection lithography is roughly equal to the wavelength of the exposure source.

The action of light on a photoresist either increases or decreases the resist solubility depending on whether it is a positive or negative photoresist, respectively. Thus, for a positive-tone photoresist, the opaque pattern on the mask will determine the features remaining in the resist layer after development (see Fig. 2). Conversely, after development of a negative photoresist, the clear pattern of the mask determines the remaining photoresist features (Fig. 2). The profile of the photoresist side walls (see Fig. 3) is critical to many applications such as patterning of hard-to-etch metals (lift-off) and mold fabrication. Figure 4 illustrates the use of a lift-off profile in the lift-off process. The resist wall profile can be controlled by adjusting resist tone, exposure dose, developer strength, and development time, as well as by other means. 


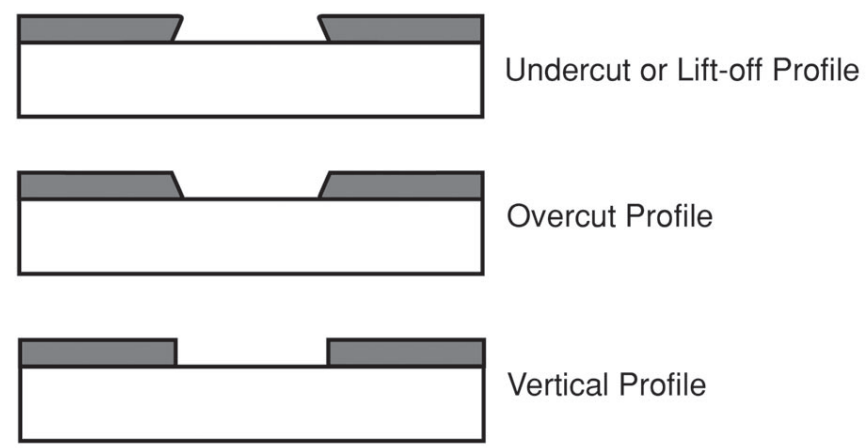

Fig. 3. The three different photoresist profiles. The undercut (lift-off) profile is used mostly in the patterning of metals in a process called "lift-off" (see Fig. 4). The overcut profile is the profile that is normally obtained from a positive-tone photoresist. The vertical profile achieves the best pattern fidelity, but is relatively difficult to obtain.

Post-exposure treatment is often desired because the reactions initiated during exposure might not have run to completion. To halt the reactions or to induce new ones, several post-exposure treatments can be used: postexposure baking, flood exposure with other types of radiation, treatment with reactive gas, and vacuum treatment.

\subsection{Development, Descumming, and Postbaking}

During the development process, selective dissolving of resist takes place (see Fig. 1D). Development can be done using a liquid (wet development), a gas, or plasma (dry development). Positive resists are typically developed in aqueous alkaline solutions (e.g., tetramethyl ammonium hydroxide), and negative resists in organic solvents.

Unwanted residual photoresist sometimes remains after development. Descumming is a procedure for removing this unwanted photoresist with a mild plasma treatment. In this process, highly energetic oxygen ions react and essentially burn away the unwanted photoresist.

Postbaking or hard baking removes residual solvents and anneals the film to promote interfacial adhesion of the resist that has been weakened either by developer penetration along the resist/substrate interface or by swelling of the resist (mainly for negative resists). Hard baking also improves the hardness of the film. Improved hardness increases the resistance of the resist to subsequent etching and deposition steps. Postbaking is usually done at higher temperatures $\left(120^{\circ} \mathrm{C}\right)$ and for longer times (e.g., $\left.20 \mathrm{~min}\right)$ than soft baking or prebaking. 


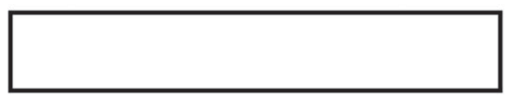

Starting Si Substrate

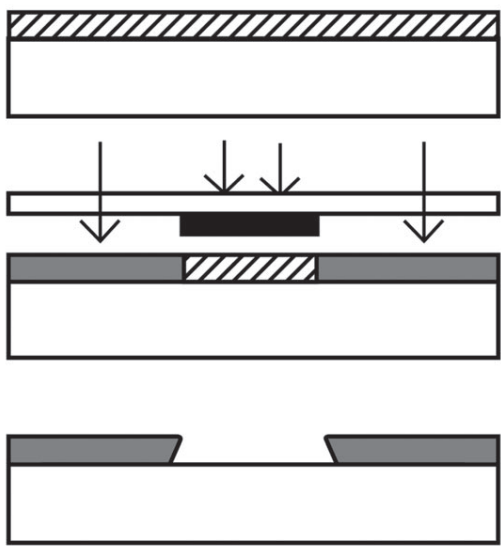

Apply Negative Photoresist

Mask Alignment and UV Exposure

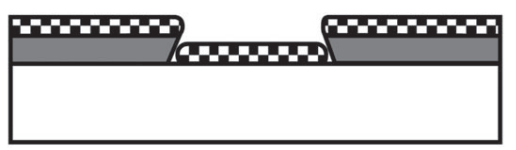

Lift-off Profile after Development

Sputter Metal

QIIIIIID

Remove Resist

Fig. 4. Example of lift-off sequence using negative resist as sacrificial layer. This method is used in cases in which the metal is difficult to etch (e.g., gold or platinum).

\subsection{Pattern Transfer}

In cases in which the photoresist is a permanent part of the final device (e.g., microfluidics, carbon MEMS [C-MEMS]), further processing may not be necessary. In most other cases, the sacrificial photoresist pattern is used as a mask for etching (subtractive) or deposition (additive) on the underlying substrate (a subtractive process; see Fig. 1E). In a subtractive process, the resist acts as a protective barrier to the etching agent, which can be a liquid solution, a gas, or plasma. After pattern transfer, the resist can be removed for further process steps. Similarly, pattern transfer can involve a deposition technique: chemical vapor deposition or e-beam evaporation. 


\section{Soft Lithography}

Soft lithography (2-4) techniques incorporate an imprint step, in which the topography of a template defines patterns created on a substrate. In soft lithography, a patterned elastomer is used as a stamp, mold, or mask to generate micropatterns and microstructures instead of using a rigid photomask. These methods include replica molding, micro-contact printing, micromolding in capillaries, and micro-transfer molding (5). The major advantage of soft lithography is its short turnaround time; it is possible to go from design to production of replicated structures in $<24 \mathrm{~h}$. The method is low in cost and, unlike photolithography, soft lithography is applicable to almost all polymers and, thus, many materials that can be prepared from polymeric precursors. Because soft materials are used, deformation of the stamp or mold, low reproducibility (owing to distortion), and defects (yield) are problems that prevent this technology from being a viable manufacturing technique, but it is widely used in research settings (2).

An example of soft lithography frequently used in creating microfluidics is polydimethylsiloxane (PDMS) molding. In this method, high-aspect-ratio microfluidics can be fabricated using SU-8 negative photoresist (MicroChem, Newton, MA) molds. SU-8 is a chemically amplified negative photoresist with high transparency. The high transparency allows light to penetrate through thick layers of photoresist, thus creating near vertical sidewall profiles. As a form of soft lithography, SU-8 structures have been used as molds for microfluidic applications. In these applications, PDMS is first poured onto the patterned SU-8 mold. The PDMS is cured, removed, and then pressed or bonded onto a flat substrate to create microchannels. Figure 5 shows a scanning electron microscopy (SEM) micrograph of a PDMS microchannel for a microfluidic system.

\section{MicroElectroMechanical Systems (MEMS)}

MEMS, or the science of miniaturization, refers to a class of devices that have at least one of their dimensions in the micrometer range. Whereas IC devices involve only electrical components (transistors, diodes, capacitors, and so on), MEMS devices take advantage of a wide range of other phenomena from mechanical to biological (BioMEMS). The materials and fabrication methods used in MEMS are much more varied than those used for IC fabrication (in which one deals principally with $\mathrm{Si}$, oxides, and metals patterned using photolithography). In contrast to the IC industry, in which the devices are carefully packaged and protected from the environment, MEMS devices, such as pressure or glucose sensors, often must have surfaces that are directly exposed to the environment in which they are sensing. Examples of successfully com- 


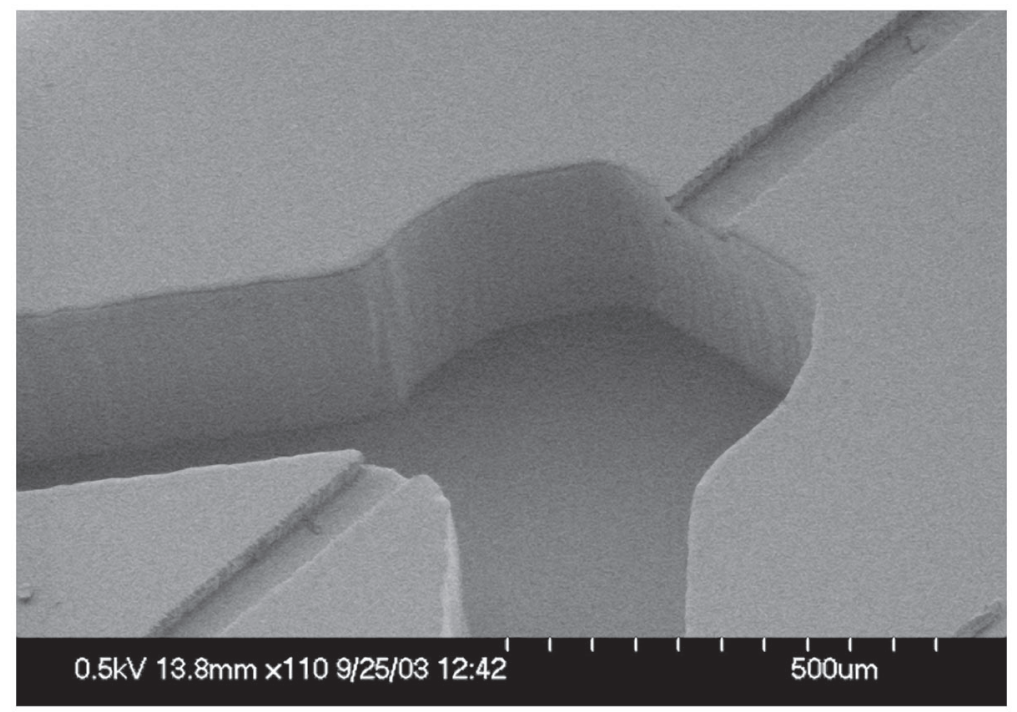

Fig. 5. SEM micrograph showing a PDMS microchannel used for hybridization of DNA on a microfluidic platform. (Courtesy of Guangyao Jia, Madou Research Group, UCI.)

mercialized mechanical devices are accelerometers, gyroscopes, tilt meters, membrane pressure sensors, micromirrors, optical MEMS switches, and inkjet printheads. Commercial BioMEMS devices include glucose sensors, lab-onchip systems, and DNA arrays. Because of the irreversible chemical reactions involved and contamination considerations, BioMEMS devices tend to be disposable.

\section{Nontraditional Materials}

Different materials that are being used or investigated for use in MEMS processes include polymers, ceramics, nitinol (shape memory alloys), biomaterials, and carbon. The Madou research group at UCI (see http:// mmadou.eng.uci.edu) has been actively pursuing research in C-MEMS derived from pyrolyzed photoresist. Patterned SU-8 photoresist is converted into carbon electrodes by subjecting the photoresist to high temperatures in an inert environment in a process called pyrolysis. C-MEMS electrodes can be easily patterned into complex three-dimensional geometries that were previously difficult or expensive to fabricate using conventional carbon electrode fabrication methods. In addition, the electrochemical properties of carbon make it an excellent electrode material (6). High-aspect-ratio carbon structures (see Fig. 6) can be obtained from SU-8 photoresist structures (see Fig. 7). These high-aspect- 


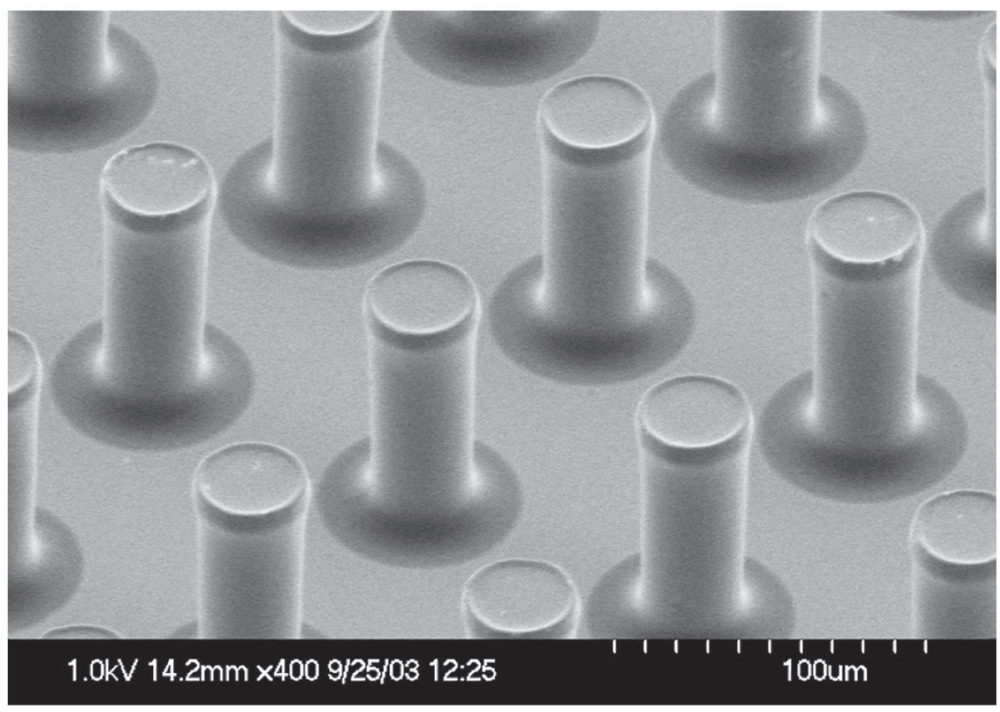

Fig. 6. SEM micrograph of carbon (C-MEMS) posts obtained by pyrolyzing SU-8 negative photoresist. (Courtesy of Chunlei Wang, Madou Research Group, UCI.)

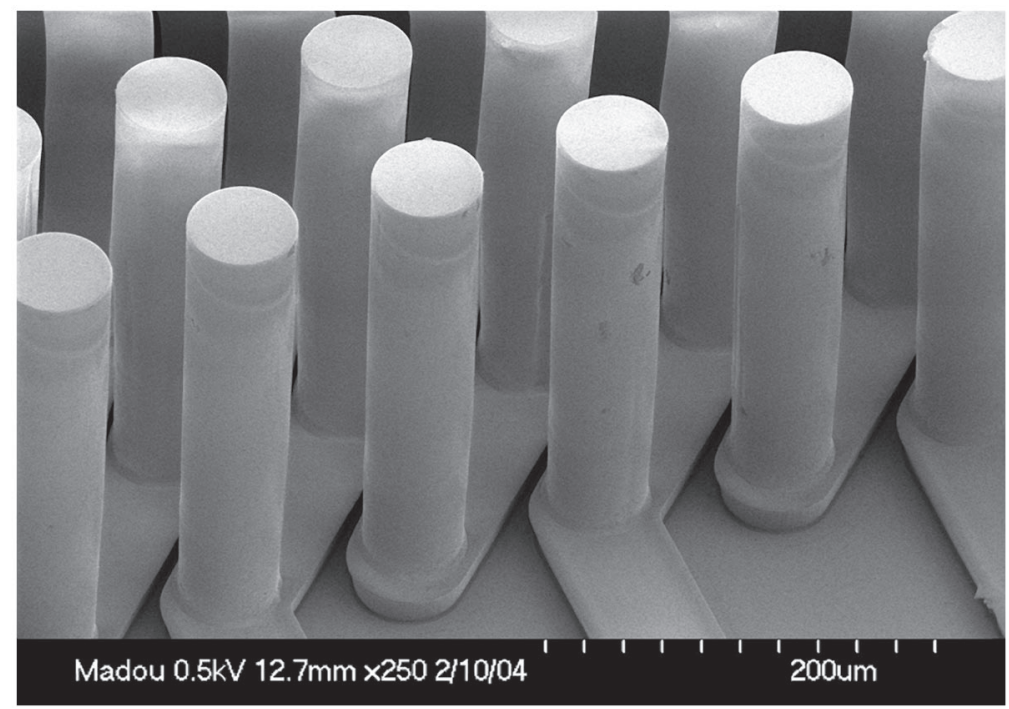

Fig. 7. SEM micrograph of a high-aspect-ratio SU-8 negative photoresist structure taken before structure was pyrolyzed into carbon. The resulting electrodes and interconnects were used for battery research. (Courtesy of Chunlei Wang, Madou Research Group, UCI.) 
ratio carbon structures are being investigated for their potential use in batteries, and chemical and biosensing applications.

\section{Conclusion}

In this chapter, we introduced lithography as an important tool for microfabrication. The application domain of lithography has greatly expanded beyond the IC world to encompass fabrication of mechanical and biological devices. The fact that some DNA arrays are nowadays made through lithography illustrates this point. With the advent of MEMS, different materials and high-aspect-ratio fabrication techniques have also been introduced. The field of microfabrication is so vast that it would be impossible to give a comprehensive review in the context of one short chapter. The reader is therefore referred to textbooks $(7,8)$ and recent reviews for a more thorough investigation.

\section{References}

1. Compton. (1994) Compton's Interactive Encyclopedia (Interactive Multimedia), Compton's New Media, Carlsbad, CA.

2. Xia, Y. and Whitesides, G. M. (1998) Soft lithography. Annu. Rev. Mater. Sci. 28, $153-184$.

3. Kumar, A. and Whitesides, G. M. (1993) Features of gold having micrometer to centimeter dimensions can be formed through a combination of stamping with an elastomeric stamp and an alkanethiol 'ink' followed by chemical etching. Appl. Phys. Lett. 63, 2002-2004.

4. Kumar, A., Abbot, N. L., Kim, E., Biebuyck, H., and Whitesiden, G. M. (1995) Patterned self-assembled monolayers and meso-scale phenomena. Acc. Chem. Res. 28, 219-226.

5. Xia, Y., Rogers, J. A., Paul, K. E., and Whitesides, G. A. (1999) Unconventional methods for fabricating and patterning nanostructures. Chem. Rev. 99, 1823-1848.

6. Kinoshita, K. (1988) Carbon, Electrochemical and Physicochemical Properties, John Wiley and Sons, New York.

7. Madou, M. J. (2002) Fundamentals of Microfabrication: The Science of Miniaturization, 2nd ed., CRC Press, Boca Raton, FL.

8. Gad-el-Hak, M. (2002) The MEMS Handbook, CRC Press, Boca Raton, FL. 
